\title{
Evaluation of Coagulation Parameters in Dogs with Overweight or Obesity
}

\author{
Amanda Dela Antonio Barbosa, Natalí Faria Martins, Samira Arruda Rosário, Paola Caroline da Silva Nunes, \\ Danielle Passarelli \& Deise Carla Almeida Leite-Dellova
}

\begin{abstract}
Background: Hemostatic alterations have already been well characterized in humans with body fat excess, being included in the list of obesity related comorbidities. Overweight and obesity are common in dogs; however, there is little information about the blood coagulation parameters in dogs with these conditions. The aim of this study was to compare hematological and coagulation parameters between lean dogs and overweight or obese dogs, including platelets count; prothrombin time (PT); activated partial thromboplastin time (aPTT), coagulation time (CT) and plasma fibrinogen concentration.

Materials, Methods \& Results: A total of 22 dogs (aged 1 to 10 years, neutered) were evaluated, 10 of them presented ideal body condition score (BCS 4-5) and formed the group 1 (control) and 12 were overweight or obese (BCS 7-9) and formed the group 2. The dogs were submitted to clinical evaluation and then to blood collection for the following laboratory tests: blood count (performed on automatic analyzer), quantification of plasma proteins by refractometry, determination PT, aPTT and plasma fibrinogen concentration using specific commercial kits, and CT by Lee-White method. Compared to group 1, group 2 presented a lower leukocytes and lymphocytes counts $(P<0.05)$ and a higher concentration of plasma fibrinogen $(P=0,026)$, but compatible with reference values. No difference was observed in the erythrogram, platelets count, total plasma protein concentration, PT, aPTT and CT between the groups. BCS was negatively correlated with leukocytes $(r=-0.45)$ and lymphocytes $(r=-0.60)$ counts and positively with plasma fibrinogen concentration $(r=+0.56)$. Discussion: The reduction in lymphocytes led to a lower leukocytes count in the dogs of group 2 . The migration of peripheral blood lymphocytes to adipose tissue has been reported in the early phase of the inflammatory process induced by obesity and could justify the reduction of circulating lymphocytes in overweight or obese dogs evaluated in this study; however, cytopathological assessment of adipose tissue was not performed. Fibrinogen has to be converted to fibrin for clot formation, so the concentration of this plasma protein is an important parameter for the evaluation of hemostasis. However, there are reports of increase in plasma fibrinogen as a result of infammatory processes. In group 2, formed by dogs with overweight or obese, the higher value of plasm fibrinogen concentration, associated with lower lymphocyte count, may suggest an early-stage inflammatory process. Similar results were described in obese humans, but also evidenced in obese dogs. Supporting this suggestion, the correlation analysis indicates that the higher the body fat excess (estimated by the BCS), the higher the fibrinogen concentration and the lower the lymphocyte count in evaluated dogs. Despite the higher concentration of plasma fibrinogen in group 2, no change was observed in the hemostasis of overweight or obese dogs, due to the normal values for platelets count, PT, aPTT and CT, excluding a hypercoagulability condition as already hypothesized by other authors for dogs and obese humans. The difference between our findings and the literature may be in the time of evolution of the disease, since we evaluated younger dogs. In conclusion, the body fat excess did not alter the erythrogram and the activity of the clotting factors, estimated by PT, aPTT and CT, but it interfered in the leukogram and increased the plasma concentration of fibrinogen in the evaluated dogs.
\end{abstract}

Keywords: body fat excess, coagulation pathways, small animals, fibrinogen. 


\section{INTRODUCTION}

According to the Global Pet Obesity Initiative Position Statement, obesity in dogs is defined as $30 \%$ above ideal body weight [29]. There is also the distinction between being overweight or being obese, established by the body condition score (BCS) scale for dogs [13].

The excessive food intake and/or inadequate energy use are among the main causes of obesity in dogs [16]. In addition, some breeds of dogs are more predisposed to weight gain, such as labrador retriever, golden retriever and pug [3,7,25].

Obesity is increasingly present in veterinary medicine, mainly, because the animals share the same unhealthy lifestyle of their tutors, characterized by physical inactivity and easy access to palatable and calorie foods [21,22].

Currently, canine obesity is considered a disease [4], which reduces quality and life expectancy [23], due to several comorbidities, e.g. dyslipidemia (which increase the risk of pancreatitis) [30], diabetes mellitus, orthopedic diseases, mammary and urinary tract neoplasias. In obese human, changes in blood coagulation were well characterized and include enhanced of platelet activation [1], increased activity of plasma coagulation factors and impaired fibrinolysis, with thromboembolism risk [9]. A single study reported the increased platelet count and reduction in aPTT in overweight and obese dogs [20]; however, little is known about the coagulation status in dogs with this condition.

The aim of this study was to compare hematological and coagulation parameters between lean dogs and overweight or obese dogs, including platelet count, PT, aPTT, CT and plasma fibrinogen concentration.

\section{MATERIALS AND METHODS}

Animals

Twenty-two dogs (12 females and 10 males), aged 1 to 10 years, neutered and of different breeds were evaluated. These dogs were selected in the Small Animal Service of the Veterinary Hospital of instituition. During the anamnesis, the tutors did not report previous diseases and the dogs presented good general condition.

The dogs were divided into two groups, according to the BCS [13]: group 1, formed by $10 \mathrm{dogs}$ (6 males and 4 females) with BCS 4-5, considered ideal, and group 2, formed by 12 dogs ( 4 males and 8 females) with BCS 7-9, i.e. in overweight or obesity.

The dogs were submitted to clinical and laboratory evaluation and only those with normal results for physical exam and laboratory tests or results consistent with excess body fat were included in this study. Obese dogs with suspected or definitive diagnosis of metabolic diseases (such as diabetes mellitus, hyperadrenocorticism or hypothyroidism) were not included. So, the justification for overweight or obesity in BCS 7-9 group was ad libitum feeding.

\section{Blood sample and laboratory tests}

After $8 \mathrm{~h}$ fasting, blood samples were performed by jugular venipuncture. Then, the blood of each dog was transferred to tube $\left.{ }^{1}: 1\right)$ with anticoagulant (ethylenediamine tetraacetic acid - EDTA) to perform the blood count, 2) containing sodium citrate for determination of PT, aPTT and plasma fibrinogen concentration, using commercial kits ${ }^{2}$ and following the manufacturer's instructions, and 3) without anticoagulant to determine CT by Lee-White method [14].

An automatic blood analyzer (BC 2800 VET $)^{3}$ was used in blood count, but the differential leukocytes counting was done manually, from blood smears [28]. Total plasma protein was measured by refractometry ${ }^{4}$.

PT, aPTT and fibrinogen were determined by the clot formation method [24], from the addition of specific reagents in the plasma sample. The kits PT (ref. 504) $)^{2}$ and aPTT (ref. 502) ) $^{2}$ contained thromboplastin plus calcium chloride or ellagic acid plus phospholipids and calcium chloride, respectively. These reagents triggered the sequential activation of clotting factors, resulting in activated thrombin and conversion of fribrinogen to fibrin, evidenced by the appearance of the clot. PT and aPTT corresponded to time (in seconds) from the addition of reagents to visualization of the clot. In the fibrinogen kit (ref. 506) ${ }^{2}$, the reagent was thrombin, so, the higher the fibrinogen concentration in plasma sample, the faster the clot was formed. The time to clot formation was converted to fibrinogen concentration $(\mathrm{mg} / \mathrm{dL})$ using a calibration curve. CT corresponded to time (in minutes) since the blood penetrated in the syringe, until the clot was formed in the glass tube [14].

\section{Statistical analysis}

Data are presented as mean values \pm standard error of the individual groups means. The results were 
submitted to analysis of variance by $\mathrm{F}$ test and the mean values of groups 1 and 2 were compared by unpaired $\mathrm{t}$ test, using PRISM package ${ }^{5}(P<0.05)$. Correlations were evaluated by the Pearson coefficient.

\section{RESULTS}

The mean values of age did not differ between groups $(P=0.172)$. The group 2 had a higher mean value for body weight and BCS. Breeds, individual and mean values of age, body weight and BCS of dogs from both groups are shown in Table 1.

All dogs presented normal values for the blood count and total plasma protein concentration [28]. Comparing the mean values, the total number of leukocytes and lymphocytes was lower in group 2 (Table 2).

No differences were found between groups for mean values of PT, aPTT and CT (Table 3 ) and the results were similar to described by other authors [5,24]. Fibrinogen concentration was higher in group 2 (254.4 \pm 17.00 versus $190.7 \pm 20.65 ; P=0.026$ ) (Figure 1 ) but did not exceed the reference values (100 - $500 \mathrm{mg} / \mathrm{dL}$ ) [28].

BCS was negatively correlated with number of leukocytes $(\mathrm{r}=-0.45 ; P=0.037)$ and lymphocytes $(\mathrm{r}=$ -0.60; $P=0.003)$. Positive and significant correlation was observed between BCS and plasma fibrinogen concentration $(\mathrm{r}=+0.56 ; P=0.007)$. Correlations between BCS and PT, aPTT and CT were weak and not significant.

\section{DISCUSSION}

Recent study has reported that factors such as age, neuter status and breed influence weight gain in dogs [8]. Increase in sedentary behavior [17], reduction of metabolic rate [2], composition and function of adipose tissue [26] and genetic predisposition [27] justify obesity in older dogs, neutered and some breeds.

In the present study, group 2 was initially formed and all dogs in this group were neutered and presented overweight or obese; agreeing with epidemiological studies that evaluated the canine obesity $[2,6,8]$. Then, to compose group 1 , which would represent the control situation, dogs were recruited with ideal BCS and also neutered. The females neutered predisposition to obesity certainly influenced in the percentage of females in group 2(67\%) [15].

The mean age value was numerically higher in group 2, but did not differ statistically from group 1 , because $67 \%$ of dogs in group 2 were aged 1 to 5 years. In a study of international scope, the researchers also observed obesity in dogs from 1-year-old [6]. In the present study, young adult dogs presented excess body fat, compatible with overweight or obesity, possibly, due to early neutered associated with ad libitum feeding. A reduced calorie diet is indicated after early neutered to decrease the risk of obesity in dogs [15].

Group 2 was composed of dogs of large breeds (33.3\%), with overweight $(16.7 \%)$ or obesity $(83.3 \%)$; therefore, mean values of body weight and BCS were higher, compared to group $1(P<0.05)$.

Dogs of group 2 did not present alterations in blood count (erythrogram and leukogram) and total plasma protein concentration, in relation to reference values [28]; but, in comparison to group 1, the lymphocytes count was lower, also reducing the total leukocytes count. In addition, a negative correlation was found between BCS and count of leukocytes and lymphocytes. The excess body fat in the dogs evaluated may have led to the reduction of circulating lymphocytes, interfering with the total leukocytes count. In humans and mice, the migration of peripheral blood lymphocytes to adipose tissue has been reported in the early phase of the inflammatory process induced by obesity [10]; however, cytopathological assessment of adipose tissue was not performed in this study.

In accordance with present results, the reduction of circulating lymphocytes in overweight and obese dogs was reported in a previous research [20]. However, these authors did not find differences in total leukocytes count but evidenced an increase in platelets count in the group of overweight and obese

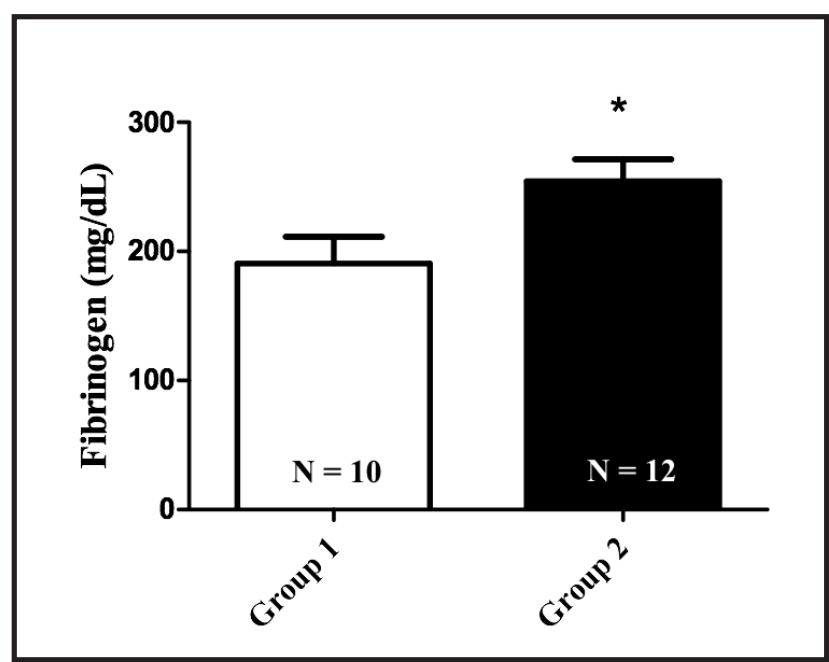

Figure 1. Concentration of plasma fibrinogen in dogs. Group 2, formed by dogs with body condition score between 7-9, had a higher average fibrinogen concentration than dogs of group 1, with body condition score between $4-5$. ${ }^{*} P=0.026$ versus group 1 . $\mathrm{N}=$ number of dogs in each group. 
Table 1. Description of breeds, individual and mean values of age, body weight and body condition score (BCS) of selected dogs.

\begin{tabular}{|c|c|c|c|c|c|c|c|c|}
\hline \multirow[b]{2}{*}{ Dog } & \multicolumn{4}{|c|}{ Group $1(\mathrm{~N}=10)$} & \multicolumn{4}{|c|}{ Group $2(\mathrm{~N}=12)$} \\
\hline & Breed & Age (years) & $\begin{array}{l}\text { Body weight } \\
\text { (Kg) }\end{array}$ & BCS & Breeds & Age (years) & $\begin{array}{l}\text { Body weight } \\
\text { (Kg) }\end{array}$ & $\mathrm{BCS}$ \\
\hline 1 & $\mathrm{Bc}$ & 1 & 17.4 & 5 & Bmd & 3 & 55.0 & 9 \\
\hline 2 & $\mathrm{Mb}$ & 1 & 7.4 & 4 & $\mathrm{~Eb}$ & 1 & 27.2 & 9 \\
\hline 3 & $\mathrm{Mb}$ & 1 & 6.3 & 5 & $\mathrm{Gr}$ & 5 & 35.0 & 9 \\
\hline 4 & $\mathrm{Mb}$ & 1 & 14.5 & 5 & Pitbull & 3 & 23.3 & 7 \\
\hline 5 & $\mathrm{Mb}$ & 2 & 11.6 & 5 & Pitbull & 5 & 30.0 & 8 \\
\hline 6 & $\mathrm{Mb}$ & 4 & 18.0 & 5 & Pug & 1 & 7.9 & 8 \\
\hline 7 & $\mathrm{Mb}$ & 5 & 12.4 & 5 & $\mathrm{Mb}$ & 1 & 14.5 & 7 \\
\hline 8 & $\mathrm{Mb}$ & 5 & 15.3 & 5 & $\mathrm{Mb}$ & 4 & 15.2 & 8 \\
\hline 9 & $\mathrm{Mb}$ & 8 & 15.6 & 4 & $\mathrm{Mb}$ & 6 & 24.4 & 9 \\
\hline 10 & Shitzu & 1 & 4.7 & 5 & $\mathrm{Mb}$ & 8 & 15.0 & 9 \\
\hline 11 & --- & --- & --- & --- & $\mathrm{Mb}$ & 8 & 19.6 & 8 \\
\hline 12 & --- & --- & --- & --- & $\mathrm{Mb}$ & 10 & 16.2 & 8 \\
\hline Mean $\pm \mathrm{SE}$ & --- & $2.90 \pm 0.78$ & $12.32 \pm 1.50$ & $4.80 \pm 0.13$ & -- & $4.58 \pm 0.87$ & $23.60 \pm 3.60^{*}$ & $8.25 \pm 0.22 *$ \\
\hline
\end{tabular}

$\mathrm{N}=$ number of dogs in each group. $\mathrm{Bc}=$ Border collie. $\mathrm{Mb}=$ Mixed breed. Bmd= Bernese mountain dog. Eb= English bulldog. Gr= Golden retriever. $\mathrm{SE}=$ standard error. $* P<0.05$ versus group 1 .

Table 2. Mean values of blood count and total plasma protein concentration of groups 1 and 2.

\begin{tabular}{cccc}
\hline Parameter & Group 1 $(\mathrm{N}=10)$ & Group 2 $(\mathrm{N}=12)$ & $P$ value \\
\hline Erythrocytes $(/ \mu \mathrm{L})$ & $6.75 \pm 0.27$ & $6.45 \pm 0.21$ & 0.388 \\
Hematocrit $(\%)$ & $49.89 \pm 1.93$ & $46.83 \pm 1.78$ & 0.258 \\
Hemoglobin $(\mathrm{g} / \mathrm{dL})$ & $16.64 \pm 0.68$ & $15.53 \pm 0.64$ & 0.249 \\
MCV $(\mathrm{fL})$ & $73.98 \pm 0.46$ & $72.50 \pm 0.77$ & 0.130 \\
MCH $(\mathrm{pg})$ & $24.65 \pm 0.26$ & $24.00 \pm 0.31$ & 0.129 \\
MCHC $(\%)$ & $33.33 \pm 0.24$ & $33.09 \pm 0.20$ & 0.446 \\
Platelets $(/ \mu \mathrm{L}) \mathrm{x} 10^{3}$ & $275.60 \pm 24.73$ & $319.20 \pm 23.97$ & 0.223 \\
Leukocytes $(/ \mu \mathrm{L})$ & $9.610 \pm 827.90$ & $7.567 \pm 418.90$ & 0.031 \\
Segmented $(/ \mu \mathrm{L})$ & $5.328 \pm 590.50$ & $4.972 \pm 626.80$ & 0.576 \\
Eosinophils $(/ \mu \mathrm{L})$ & $666.50 \pm 127.90$ & $533.20 \pm 92.36$ & 0.398 \\
Lymphocytes $(/ \mu \mathrm{L})$ & $3.156 \pm 394.70$ & $1.740 \pm 136.10$ & 0.002 \\
Monocytes $(/ \mu \mathrm{L})$ & $449.30 \pm 77.78$ & $322.40 \pm 60.93$ & 0.207 \\
Total plasma protein $(\mathrm{g} / \mathrm{dL})$ & $6.72 \pm 0.14$ & $7.05 \pm 0.31$ & 0.375 \\
\hline
\end{tabular}

$\mathrm{N}=$ number of dogs in each group. $\mathrm{MCV}=$ mean corpuscular volume; $\mathrm{MCH}=$ mean corpuscular hemoglobin; $\mathrm{MCHC}=$ medium corpuscular hemoglobin concentration.

Table 3. Mean values of prothrombin time (PT), activated partial thromboplastin time (aPTT) and coagulation time (CT) of groups 1 and 2.

\begin{tabular}{cccc}
\hline Parameter & Group 1 $(\mathrm{N}=10)$ & Group 2 $(\mathrm{N}=12)$ & $P$ value \\
\hline PT (s) & $14.10 \pm 0.530$ & $14.54 \pm 0.892$ & 0.692 \\
aPTT (s) & $25.27 \pm 0.546$ & $25.00 \pm 0.903$ & 0.815 \\
CT (min) & $3.80 \pm 0.239$ & $3.72 \pm 0.285$ & 0.819 \\
\hline
\end{tabular}


dogs. In another research, dogs with $\mathrm{BCS} \geq 7$ had the leukocytes count and plasma protein concentrations more elavated than dogs with BCS 4-6 [19].

The dogs of group 1 and 2 did not present alterations in coagulogram, due to normal values for platelets, PT, aPTT, CT and fibrinogen. PT evaluates the activity of factors VII, X, V, II and the extrinsic and common pathways, and aPTT, the activity of factors XII, XI, IX, VIII, X, and the intrinsic and common pathways of coagulation [5]. Moreover, fibrinogen (or factor I) is part of the common pathway and CT evaluates the intrinsic and common pathways of coagulation [24].

A recent study showed a reduced aPTT, without difference in PT and fibrinogen concentration, in overweight and obese dogs, when compared to lean dogs [20]. In the present study, the dogs of group 2 presented PT, aPTT and CT similar to group 1; however, the fibrinogen concentration was higher $(P<0.05)$. Besides that, a moderate and positive correlation was verified between BCS and plasma fibrinogen concentration in the evaluated dogs.

Alterations in leukocytes [19] and the coagulogram [20] of overweight or obese dogs were related to a low intensity inflammatory state, evidenced by the increase of inflammatory markers, such as interleukin 6 and c reactive protein, in serum of dogs [20]. Plasma fibrinogen is also an inflammatory marker, which may be increased in obese dogs [31].

There are reports that obese humans with metabolic syndrome, characterized by the simultaneous occurrence of insulin resistance and pro-inflammatory status, showed an increase in the concentration of coagulation factors, including fibrinogen, and decreased in fibrinolysis. All these changes lead to a state of hypercoagulability, with high risk of thromboembolism and cardiovascular complications [11,12,18].

The hypothesis of a hypercoagulable state in overweight and obese dogs was raised by other authors, due to increased platelets, aPTT reduction, increased activity of some clotting factors (VII and X) and of inflammation markers [20]. However, this was not evidenced in the present study.

In group 2, the higher value of plasm fibrinogen concentration, associated with lower lymphocyte count, may suggest an early-stage inflammatory process, even in dogs in good condition. Obese dogs evaluated in the present study were younger (up to 10 years old) than obese dogs assessed in previous studies (up to 15 years old) $[19,20]$. It is possible that the excess of body fat has not yet caused an inflammatory process as intense as that observed by these authors $[19,20]$. With the maintenance of the disease and over time, more evident leukocytes and coagulogram alterations could be observed, besides the increase of plasma fibrinogen. In agreement with this possibility, the increase of plasma fibrinogen, with normal reactive protein, was observed in obese Beagles of up to 10 years of age, suggesting a mild inflammation [31]. However, the consequences of increased fibrinogen in obese dogs are not yet well established.

\section{CONCLUSION}

The accumulation of body fat did not alter the erythrogram and the activity of the clotting factors, estimated by PT, aPTT and CT, but it interfered in the leukogram and increased the plasma concentration of fibrinogen in the evaluated dogs.

\section{MANUFACTURERS}

${ }^{1}$ Labor Import Comercial Importadora Exportadora. Osasco, SP, Brazil.

${ }^{2}$ Labtest Diagnóstica S.A. Lagoa Santa, MG, Brazil.

${ }^{3}$ Mindray BioMedical Electronics Co. Ltd. Shenzhen, China.

${ }^{4}$ Quimis Aparelhos Científicos. Diadema, SP, Brazil.

${ }^{5} \mathrm{GraphPad}$ Software Inc. San Diego, CA, USA.

Ethical approval. This experiment was approved by Ethics Committee for Animal Use of Faculty of Animal Science and Food Engineering of the University of São Paulo (protocol number: 5400240816).

Declaration of interest. The authors report no conflicts of interest. The authors alone are responsible for the content and writing of the paper. 


\section{REFERENCES}

1 Anfossi G., Russo I. \& Trovati M. 2009. Platelet dysfunction in central obesity. Nutrition, Metabolism and Cardiovascular Diseases. 19(6): 440-449.

2 Aptekmann K.P., Suhett W.G., Mendes Junior A.F., Souza G.B., Tristão A.P.P.A., Adams F.K., Aoki G.D., Palacios Junior R.J.G., Carciofi A.C \& Tinucci-Costa M. 2014. Aspectos nutricionais e ambientais da obesidade canina. Ciência Rural. 44(11): 2039-2044.

3 Bland I.M., Guthrie-Jones A., Taylor R.D. \& Hill J. 2009. Dog obesity: owner attitudes and behaviour. Preventive Veterinary Medicine. 92: 333-340.

4 Burns K. 2018. Taking on obesity as a disease: Statement, sessions, and toolkit address the excess weight so common now in cats, dogs, and horses. [Fonte:<https://www.avma.org/News/JAVMANews/Pages/181001a.aspx $>$ ]. [Accessed online in June 2018].

5 Dos Anjos Lopes S.T., Emanuelli M.P., Schmidt C., Raiser A.G., Mazzanti A. \& Alves A.S. 2005. Valores de referência do tempo de protrombina (TP) e tempo de tromboplastina parcial ativada (TTPa) em cães. Ciência Rural. 35(2): 381-384.

6 Flanagan J., Bissot T., Hours M.A., Moreno B., Feugier A. \& German A.J. 2017. Success of a weight loss plan for overweight dogs: The results of an international weight loss study. PloS ONE. 12(9): e0184199.

7 German A.J. 2006. The growing problem of obesity in dogs and cats. The Journal of Nutrition. 136: 1940S-1946S.

8 German A.J., Blackwell E., Evans M. \& Westgarth C. 2017. Overweight dogs exercise less frequently and for shorter periods: results of a large online survey of dog owners from the UK. Journal of Nutritional Science. 6: 1-4.

9 Kaye S.M., Pietiläinen K.H., Kotronen A., Joutsi-Korhonen L., Kaprio J., Yki-Järvinen H., Silveira A., Hamsten A., Lassila R. \& Rissanen A. 2012. Obesity related derangements of coagulation and fibrinolysis: a study of obesitydiscordant monozygotic twin pairs. Obesity. 20(1): 88-94.

10 Kintscher U., Hartge M., Hess K., Foryst-Ludwig A., Clemenz M., Wabitsch M., Fischer-Posovszky P., Barth T.F., Dragun D., Skurk T., Hauner H., Blüher M., Unger T., Wolf A.M., Knippschild U., Hombach V. \& Marx N. 2008. T-lymphocyte infiltration in visceral adipose tissue: a primary event in adipose tissue inflammation and the development of obesity-mediated insulin resistance. Arteriosclerosis, thrombosis, and vascular biology. 28(7): 13041310 .

11 Kotronem A., Joutsi-Korhonen L., Sevastianova K., Bergholm R., Hakkarainen A., Pietilainem K.H., Lundbom N., Rissanen A., Lassila R. \& Yki-Jarvinen H. 2011. Increased coagulation factor VIII, IX, XI and XII activities in non-alcoholic fatty liver disease. Liver International. 31: 176-183.

12 Kupcinskiene K., Trepenaitis D., Petereit R., Kupcinskas J., Gudaityte R., Maleckas A. \& Macas A. 2017. Monitoring of hypercoagulability by thromboelastography in bariatric surgery. Medical Science Monitor: International Medical Journal of Experimental and Clinical Research. 23: 1819-1826.

13 Laflamme D. 1997. Development and validation of a body condition score system for dogs. Canine Practice. 22: 1015.

14 Lee R.I. \& White P.D. 1913. A clinical study of the coagulation time of blood. The American Journal of the Medical Sciences. 145: 495-503.

15 McKenzie B. 2010. Evaluating the benefits and risks of neutering dogs and cats. CAB Reviews: Perspectives in Agriculture, Veterinary Science, Nutrition and Natural Resources. 5: 1-18.

16 Montoya-Alonso J.A., Bautista-Castaño I., Peña C., Suárez L., Juste M.C. \& Tvarijonaviciute A. 2017. Prevalence of canine obesity, obesity-related metabolic dysfunction, and relationship with owner obesity in an obesogenic region of spain. Frontiers in Veterinary Science. 4(59): 1-4.

17 Morrison R., Penpraze V., Greening R., Underwood T., Reilly J.J. \& Yam P.S. 2014. Correlates of objectively measured physical activity in dogs. The Veterinary Journal. 199(2): 263-267.

18 Nieuwdorp M., Stroes E.S.G., Meijers J.C.M. \& Buller H. 2005. Hypercoagulability in the metabolic syndrome. Current Opinion in Pharmacology. 5(2): 155-159.

19 Radakovich L.B., Truelove M.P., Pannone S.C., Olver C.S. \& Santangelo K.S. 2017. Clinically healthy overweight and obese dogs differ from lean controls in select CBC and serum biochemistry values. Veterinary Clinical Pathology. 46(2): 221-226. 
20 Rafaj R.B., Kuleš J., Marinculić A., Tvarijonaviciute A., Ceron J., Mihaljević Ž., Tumpa A. \& Mrljak V. 2016. Plasma markers of inflammation and hemostatic and endothelial activity in naturally overweight and obese dogs. BMC Veterinary Research. 13(1): 13.

21 Raffan E. 2013. The big problem: battling companion animal obesity. Veterinary Record. 173: 287-291.

22 Raffan E., Smith S.P., O'Rahilly S. \& Wardle J. 2015. Development, factor structure and application of the Dog Obesity Risk and Appetite (DORA) questionnaire. PeerJ. 3 :1-27.

23 Sandøe P., Palmer C., Corr S., Astrup A. \& Bjørnvad C.R. 2014. Canine and feline obesity: a one health perspective. Veterinary Record. 175(24): 610-616.

24 Stockham S.L.\& Scott M.A. 2011. Fundamentos de Patologia Clínica Veterinária. 2. ed. Rio de Janeiro: Guanabara Koogan, pp.213-262.

25 Such Z.R. \& German A.J. 2015. Best in show but not best shape: a photographic assessment of show dog body condition. Veterinary Record. 177(5): 125.

26 Swanson K.S., Belsito K.R., Vester B.M. \& Schook L.B. 2009. Adipose tissue gene expression profiles of healthy young adult and geriatric dogs. Archives of Animal Nutrition. 63: 160-171.

27 Switonski M. \& Mankowska M. 2013. Dog obesity-the need for identifying predisposing genetic markers. Research in Veterinary Science. 95(3): 831-836.

28 Thrall M.A. 2006. Hematologia e Bioquímica Clínica Veterinária. São Paulo: ROCA, pp.170-187.

29 Ward E., German A.J. \& Churchill J.A. 2018. The global pet obesity initiative position statement. [Fonte: <https:// static1.squarespace.com/static/597c71d3e58c621d06830e3f/t/5b54f003aa4a995f7a98fcd6/1532293123968/Global+p et+obesity+initiative+position+statement_July\%2020_2018.pdf>]. [Accessed online in July 2018].

30 Xenoulis P.G. \& Steiner J.M. 2010. Lipid metabolism and hyperlipidemia in dogs. The Veterinary Journal. 183(1): $12-21$.

31 Xu J., Bourgeois H., Vandermeulen E., Vlaeminck B., Meyer E., Demeyere K. \& Hesta M. 2015. Secreted phospholipase A2 inhibitor modulates fatty acid composition and reduces obesity-induced inflammation in Beagle dogs. The Veterinary Journal. 204(2): 214-219. 